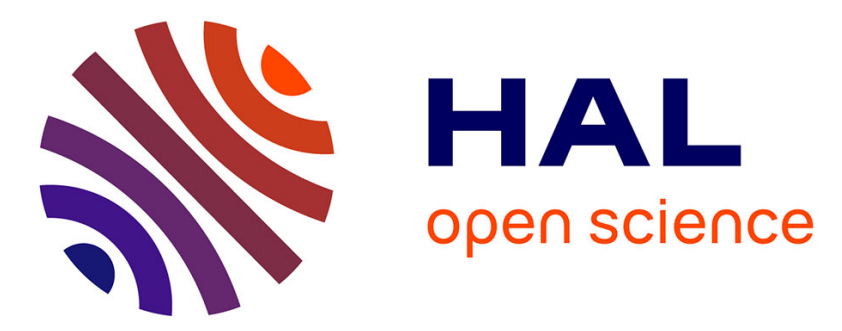

\title{
Robust high order non-conforming finite element formulation for time domain fluid-structure interaction
}

Julien Diaz, Patrick Joly

\section{To cite this version:}

Julien Diaz, Patrick Joly. Robust high order non-conforming finite element formulation for time domain fluid-structure interaction. Journal of Computational Acoustics, 2005, 13 (3), pp.403-431. 10.1142/S0218396X05002736 . inria-00409201

\section{HAL Id: inria-00409201 https://hal.inria.fr/inria-00409201}

Submitted on 19 Aug 2009

HAL is a multi-disciplinary open access archive for the deposit and dissemination of scientific research documents, whether they are published or not. The documents may come from teaching and research institutions in France or abroad, or from public or private research centers.
L'archive ouverte pluridisciplinaire HAL, est destinée au dépôt et à la diffusion de documents scientifiques de niveau recherche, publiés ou non, émanant des établissements d'enseignement et de recherche français ou étrangers, des laboratoires publics ou privés. 


\title{
ROBUST HIGH ORDER NON CONFORMING FINITE ELEMENT FORMULATION FOR TIME DOMAIN FLUID-STRUCTURE INTERACTION
}

\author{
Julien DIAZ \\ INRIA Rocquencourt - Domaine de Voluceau - 78153 Le Chesnay - France \\ julien.diaz@inria.fr \\ Patrick JOLY \\ INRIA Rocquencourt - Domaine de Voluceau - 78153 Le Chesnay - France \\ patrick.joly@inria.fr \\ Received (Day Month Year) \\ Revised (Day Month Year)
}

\begin{abstract}
In this paper we present various numerical method for solving time-dependent fluid-structure interaction problem in two or three dimensions that we claim to be efficient, robust and highly accurate. These methods, based on mixed variational formulations are explicit and conservative and can be of arbitrary high order in space. Their accuracy will be illustrated via a comparison with analytical solutions in simple configuration.
\end{abstract}

Keywords: Fluid-structure interaction; high order finite elements; non-conforming meshes; local time-stepping;

\section{Introduction}

Fluid-structure interaction problems appear in numerous and various physical applications such as :

- elastic wave propagation for seismics ${ }^{11}$ (sea bottom);

- ultra-sound waves in biological tissues, such as the bone ${ }^{7}$ :

- vibrations of solid structures (a plane, a car, a musical instrument) immersed in surrounding fluids ${ }^{10,8}$ :

An abundant literature has been devoted on the development of efficient numerical methods for solving this type of problem in the frequency domain. Much less attention has been paid to time-dependent problems that introduce additional difficulties, in particular concerning the stability of the time-stepping procedure. Moreover, one is more and more led, in scientific computing, to consider large scale problems as domain decomposition problems for which the use of independent (and non-conforming) meshes in each domain is desired. For time-dependent problems the question is the use of local time-steps.

The objective of this paper is to present various numerical methods for the treatment of time dependent fluid-structure interaction problem. These methods are in the spirit of the meth- 
ods discussed in $^{3}$ and in the continuation of several works at team ONDES at INRIA ${ }^{1,2,6,8}$. They share the following properties :

- their construction is based on (mixed) variational formulations of the complete system ;

- they remain explicit except maybe at the interface ;

- these are conservative methods : the stability is ensured through the conservation of an energy ;

- they can be of arbitrary high order in space.

The outline of the article is as follows. In section 2 we motivate the practical interest of using in this context non-conforming grids and local time-step. In section 3 we present the two main variational formulation that will constitute the basis of our methods : the so-called primal-primal and dual-dual formulations. For each formulation, in section 4, a mixed finite element approximation is proposed for the space discretization. In section 5, the crucial question of the time discretization is considered with an emphasis on the stability analysis. Finally, in section 6, we present numerical experiments that aim at validating (section 6.1) our methods and give an example of possible applications (section 6.2).

\section{Motivation of the work}

\subsection{Choice of the formulation of the problem}

It is well-known that an acoustic medium can be regarded as the limit of an isotropic elastic medium when Lamé's coefficient $\mu$ tends to zero. As a consequence, when one has a computational code for elastodynamics equations with variable coefficients, it is natural to try to treat the acoustic medium as a particular region in which $\mu$ is zero (or very small) (see for instance ${ }^{7}$ ). There are several disadvantages to this :

- for computational codes based on $H^{1}$ finite elements for the displacement field, taking $\mu=0$ makes lose the $H^{1}$ coercivity result (only the $L^{2}$ norm of the displacement field is controlled);

- for the discrete system (or equivalently $\mu$ very small) parasitic very small S-waves may be generated ;

- a full stress tensor is computed in the fluid where a single pressure is a priori needed.

That is why we shall choose to treat here the fluid-structure interaction problem as a transmission problem between a system in velocity-pressure $\left(\boldsymbol{v}_{f}, p\right)$ in the fluid and velocity-stress $\left(\boldsymbol{v}_{s}, \underline{\underline{\sigma}}\right)$ in the solid as it is done for instance in ${ }^{9}$.

\subsection{Interest of non-conforming meshes and local time-stepping}

With standard numerical methods, the choice of the discretization parameters (the space step $h$ and the time step $\Delta t$ ) is driven by the data of the experiment to be simulated. The two essential ones are the typical wavelength $\lambda$ and the wave speed $c$. Let us explain below 
how this works in practice and why one is encouraged to use different space steps and time steps in the fluid and the solid media :

(1) for the space discretization, the usual criterion is to take $h \leq \lambda / N$, where $\mathrm{N}$ is a given number of points per wavelength which depends on the considered scheme, the desired accuracy, the integration time... In a fluid structure interaction problem, at least three wavelengths have to be taken into account, namely $\lambda_{f}$ for the fluid, $\lambda_{S}$ for the shear waves and $\lambda_{P}$ for the pressure waves. Assume for simplicity that the number $\mathrm{N}$ required for the desired accuracy is the same for fluid and solid media and that one is in the typical situation where $\lambda_{f}<\lambda_{S}<\lambda_{P}$. Taking $h_{f}=\lambda_{f} / N \neq h_{s}=\lambda_{S} / N$ would ensure the same accuracy as $h_{f}=h_{s}=\lambda_{f} / N$ and would require less CPU time and memory ;

(2) for stability reasons, when one uses explicit schemes in time, as it is our objective, the time step is constrained by a so-called CFL (Courant-Friedrichs-Levy) condition :

$$
\frac{c \Delta t}{h} \leq \alpha,
$$

where $\alpha$ depends on the numerical method. On the other hand, for a lot a numerical schemes, choosing $c \Delta t / h$ too small increases needlessly the computational time and induces numerical dispersion phenomena, which could deeply affect the accuracy of the method. If one chooses the same time step in both solid and fluid media, it will satisfy the condition

$$
\Delta t \leq \min \left(\alpha_{f} \frac{h_{f}}{c_{f}}, \alpha_{s} \frac{h_{s}}{c_{P}}\right) .
$$

In a situation where $c_{f} \ll c_{P}$ (the typical ratio is one to three), we will have

$$
\Delta t=\alpha_{s} \frac{h_{s}}{c_{P}} \ll \alpha_{f} \frac{h_{f}}{c_{f}} .
$$

Therefore using different time steps $\Delta t_{f}$ and $\Delta t_{s}$ in each grid will give us some flexibility to ensure the balance of the CFL numbers in both fluid and solid media.

\subsection{About the coupling strategy between fluid and solid}

Working with non-conforming meshes in space and time, the main difficulty is reduced to the treatment of the transmission conditions. In what we shall present here, we shall privilege the robustness of the system by imposing the stability of the coupling method. To do that, two basic ingredients are the following :

- base the space discretization on a variational formulation of the coupling system ;

- ensure the stability with the conservation of some appropriate discrete energy.

As far as space discretization is concerned, the well-known mortar element method is a possible approach to deal with the special non-conformity of the problem. However, such method involves the introduction of an additional interface unknown, namely a Lagrange multiplier. 
And the discretization of this multiplier implies some constraints, linked in particular to the verification of some inf-sup condition. In this paper we shall privilege the methods that avoids the introduction of Lagrange multipliers.

\section{Obtaining the variational coupled formulation}

We denote by $d$ the dimension of the problem $(d=2$ or $d=3), \boldsymbol{x}$ the space variable, $t$ the time, by $\Omega_{f}$ the fluid medium and by $\Omega_{s}$ the solid medium. Both $\Omega_{f}$ and $\Omega_{s}$ are supposed to be open sets of $\mathbb{R}^{d}$ such that (see figure 1)

$$
\Omega_{f} \cup \Omega_{s}=\mathbb{R}^{d} \text { and } \partial \Omega_{f}=\partial \Omega_{s}=\Gamma, \text { the interface between the two media }
$$

We lastly denote by $\boldsymbol{n}$ the unit normal vector to $\Gamma$, going from $\Omega_{s}$ to $\Omega_{f}$.

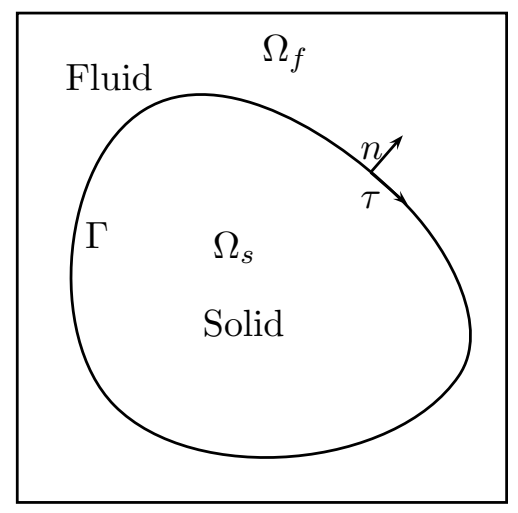

Fig. 1. Illustration of the notation

\subsection{The variational formulations for the elastic medium}

In what follows $\boldsymbol{v}_{s}=\boldsymbol{v}_{s}(\boldsymbol{x}, t)$ denotes the velocity field in $\Omega_{s}, \underline{\underline{\sigma}}=\underline{\underline{\sigma}}(\boldsymbol{x}, t)$ the stress tensor, $\rho_{s}\left(=\rho_{s}(\boldsymbol{x})\right)>0$ the density and $\underline{\underline{C}}=\left(\left(c_{i j k l}\right)\right)(=\underline{\underline{C}}(\boldsymbol{x}))$ the elastic tensor that satisfy the usual symmetry and positivity relations, almost everywhere in $\Omega_{s}$ :

$$
c_{i j k l}=c_{j i k l}=c_{i j l k}=c_{k l i j} \quad \text { and } \quad \underline{\underline{C}} \underline{\underline{\xi}}: \xi \geq \alpha \xi: \xi \quad \text { for some } \alpha>0 .
$$

where $\underline{\underline{\sigma}}: \underline{\underline{\xi}}$ denotes the usual scalar products over tensors : $\underline{\underline{\sigma}}: \underline{\underline{\xi}}=\sigma_{i j} \xi_{i j}=\operatorname{tr}\left(\xi^{t} \sigma\right)$. The stress-velocity form of linear elastodynamics equations in $\Omega_{s}$ is :

$$
(\mathrm{S}) \quad\left\{\begin{array}{l}
\frac{\partial \underline{\underline{\underline{\sigma}}}}{\partial t}-\underline{\underline{C}} \underline{\underline{\varepsilon}}\left(\boldsymbol{v}_{s}\right)=0 \\
\rho_{s} \frac{\partial \boldsymbol{v}_{s}}{\partial t}-\operatorname{div} \underline{\underline{\sigma}}=0
\end{array}\right.
$$


where $\underline{\underline{\varepsilon}}\left(\boldsymbol{v}_{s}\right)=\frac{1}{2}\left(\underline{\underline{\nabla}} \boldsymbol{v}_{s}+\underline{\underline{\nabla}} \boldsymbol{v}_{s}^{t}\right)$ and $\operatorname{div}$ is the usual divergence operator for tensors.

We consider below two mixed variational formulations for $(\mathrm{S})$.

The primal formulation. It corresponds to looking for $v_{s}$ in $\boldsymbol{H}^{1}\left(\Omega_{s}\right)=\left(H^{1}\left(\Omega_{s}\right)\right)^{d}$ and $\sigma$ merely in

$$
\mathbb{L}^{2}\left(\Omega_{s}\right)=\left\{\underline{\underline{\sigma}} \mid \sigma_{i j} \in \boldsymbol{L}^{2}\left(\Omega_{s}\right)\right\} .
$$

(we do not impose a priori any symmetry conditions) :

Find $\left(\boldsymbol{v}_{s}, \underline{\underline{\sigma}}\right): t \in \mathbb{R}^{+} \mapsto \boldsymbol{H}^{1}\left(\Omega_{s}\right) \times \mathbb{L}^{2}\left(\Omega_{s}\right)$ such that for all $\left(\boldsymbol{w}_{s}, \underline{\underline{\xi}}\right) \in \boldsymbol{H}^{1}\left(\Omega_{s}\right) \times \mathbb{L}^{2}\left(\Omega_{s}\right)$,

$$
(\mathrm{SP})\left\{\begin{array}{l}
\frac{d}{d t} \int_{\Omega_{s}} \underline{\underline{\sigma}}: \underline{\underline{\xi}}-\int_{\Omega_{s}} \underline{\underline{C}} \underline{\underline{\nabla}} \boldsymbol{v}_{s}: \underline{\underline{\xi}}=0 \\
\frac{d}{d t} \int_{\Omega_{s}} \rho_{s} \boldsymbol{v}_{s} \cdot \boldsymbol{w}_{s}+\int_{\Omega_{s}} \underline{\underline{\nabla}} \boldsymbol{w}_{s}: \underline{\underline{\sigma}}-\int_{\Gamma}(\underline{\underline{\sigma} \boldsymbol{n})}) \cdot \boldsymbol{w}_{s}=0
\end{array}\right.
$$

Remark 3.1. It is worthwhile to notice that :

- the equivalence between (S) and (SP) follows from $\underline{\underline{C}} \underline{\underline{\varepsilon}}\left(\boldsymbol{v}_{s}\right)=\underline{\underline{C}} \underline{\underline{\nabla}} \boldsymbol{v}_{s}$, because of (1) ;

- at this level, we do not pay any attention, in equation $2 \mathrm{~b}$, to the boundary term which does not make sense in the prescribed functional spaces. This will be treated when taking into account the transmission conditions (see section 3.3).

The dual formulation. It corresponds to looking for $v_{s}$ merely in $\boldsymbol{L}^{2}\left(\Omega_{s}\right)=\left(L^{2}\left(\Omega_{s}\right)\right)^{d}$ and $\sigma$ in the space of symmetric $H_{d i v}$ tensor field :

$$
\mathbb{H}_{\text {div }}^{\text {sym }}\left(\Omega_{s}\right)=\left\{\underline{\underline{\sigma}} \in \mathbb{L}^{2}\left(\Omega_{s}\right) \mid \operatorname{div} \underline{\underline{\sigma}} \in \boldsymbol{L}^{2}\left(\Omega_{s}\right) \text { and } \sigma_{i j}=\sigma_{j i}\right\} .
$$

We recall here that, thanks to (1) the map $\underline{\underline{\varepsilon}} \mapsto \underline{\underline{C}} \underline{\underline{\varepsilon}}$ is invertible when restricted to symmetric tensors and we denote by $\underline{\underline{A}}$ the corresponding inverse map $(\underline{\underline{A}}$ also satisfies (1)).

$$
\begin{aligned}
& \text { Find }\left(\boldsymbol{v}_{s}, \underline{\underline{\sigma}}\right): t \in \mathbb{R}^{+} \mapsto \boldsymbol{L}^{2}\left(\Omega_{s}\right) \times \mathbb{H}_{d i v}^{\text {sym }}\left(\Omega_{s}\right) \text { such that for all }\left(\boldsymbol{w}_{s}, \underline{\underline{\xi}}\right) \in \boldsymbol{L}^{2}\left(\Omega_{s}\right) \times \mathbb{H}_{d i v}^{s y m}\left(\Omega_{s}\right), \\
& \qquad(\mathrm{SD})\left\{\begin{array}{l}
\frac{d}{d t} \int_{\Omega_{s}} \underline{\underline{A}} \underline{\underline{\sigma}}: \underline{\underline{\xi}}+\int_{\Omega_{s}} \operatorname{div} \underline{\underline{\xi}} \cdot \boldsymbol{v}_{s}-\int_{\Gamma} \underline{\underline{\xi} \boldsymbol{n})} \cdot \boldsymbol{v}=0 \\
\frac{d}{d t} \int_{\Omega_{s}} \rho_{s} \boldsymbol{v}_{s} \cdot \boldsymbol{w}_{s}-\int_{\Omega_{s}} \boldsymbol{w}_{s} \cdot \operatorname{div} \underline{\underline{\sigma}}=0
\end{array}\right.
\end{aligned}
$$

Remark 3.2. It is worthwhile to notice that : 
- to obtain equation $(4 \mathrm{a})$ we have used the fact that div is the formal adjoint of $\underline{\underline{\varepsilon}}(\cdot)$ when acting on symmetric tensors fields;

- here again, in equation (4a), the boundary term, which is not a priori well defined, will be treated in section 3.3.

\subsection{The variational formulations for the fluid medium}

In what follows $\boldsymbol{v}_{f}=\boldsymbol{v}_{f}(\boldsymbol{x}, t)$ denotes the velocity field in $\Omega_{f}, p=p(\boldsymbol{x}, t)$ the pressure field, $\rho_{f}\left(=\rho_{f}(\boldsymbol{x})\right)>0$ the density and $c_{f}\left(=c_{f}(\boldsymbol{x})\right)>0$ the sound speed.

The pressure-velocity form of the acoustic equations is :

$$
\left\{\begin{array}{l}
\frac{\partial p}{\partial t}+c_{f}^{2} \rho_{f} \operatorname{div} \boldsymbol{v}_{f}=0 \\
\rho_{f} \frac{\partial \boldsymbol{v}_{f}}{\partial t}+\nabla p=0
\end{array}\right.
$$

As for the solid, we can write two mixed variational formulations in the fluid :

\section{The primal formulation.}

Find $\left(p, \boldsymbol{v}_{f}\right): t$ in $\mathbb{R}^{+} \mapsto H^{1}\left(\Omega_{f}\right) \times \boldsymbol{L}^{2}\left(\Omega_{f}\right)$ such that for all $\left(q, w_{f}\right) \in H^{1}\left(\Omega_{f}\right) \times \boldsymbol{L}^{2}\left(\Omega_{f}\right)$,

$$
\left\{\begin{array}{l}
\frac{d}{d t} \int_{\Omega_{f}} \frac{p q}{c_{f}^{2} \rho_{f}}-\int_{\Omega_{f}} \nabla q \cdot \boldsymbol{v}_{f}-\int_{\Gamma} q\left(v_{f} \cdot \boldsymbol{n}\right)=0 \\
\frac{d}{d t} \int_{\Omega_{f}} \rho_{f} \boldsymbol{v}_{f} \cdot \boldsymbol{w}_{f}+\int_{\Omega_{f}} \boldsymbol{\nabla} p \cdot \boldsymbol{w}_{f}=0
\end{array}\right.
$$

\section{The dual formulation.}

Find $\left(p, \boldsymbol{v}_{f}\right):$ in $\mathbb{R}^{+} \mapsto L^{2}\left(\Omega_{f}\right) \times \boldsymbol{H}_{\text {div }}\left(\Omega_{f}\right)$ such that for all $\left(q, w_{f}\right) \in L^{2}\left(\Omega_{f}\right) \times \boldsymbol{H}_{\text {div }}\left(\Omega_{f}\right)$,

$$
\left\{\begin{array}{l}
\frac{d}{d t} \int_{\Omega_{f}} \frac{p q}{c_{f}^{2} \rho_{f}}+\int_{\Omega_{f}} q \operatorname{div} \boldsymbol{v}_{f}=0 \\
\frac{d}{d t} \int_{\Omega_{f}} \rho_{f} \boldsymbol{v}_{f} \cdot \boldsymbol{w}_{f}-\int_{\Omega_{f}} p \operatorname{div} \boldsymbol{w}_{f}-\int_{\Gamma} p\left(\boldsymbol{w}_{f} \cdot \boldsymbol{n}\right)=0
\end{array}\right.
$$




\subsection{The coupling between the fluid and the solid}

Let us first recall that the natural transmission conditions on the interface $\Gamma$ are the continuity of normal velocities and of normal stresses :

$$
(\mathrm{TC})\left\{\begin{array}{l}
\boldsymbol{v}_{s} \cdot \boldsymbol{n}=\boldsymbol{v}_{f} \cdot \boldsymbol{n} \\
\underline{\underline{\sigma}} \boldsymbol{n}=-p \boldsymbol{n} .
\end{array}\right.
$$

Coupling the primal formulation in one domain with the dual formulation in the other one would involved the introduction of a Lagrange multiplier as for mortar elements. The two other possibilities we are considering here are multiplier free.

The primal-primal formulation We wish to couple (SP) to (FP). We use the continuity of normal stresses condition (8b) in the boundary integral of $(3 \mathrm{~b})$ :

$$
\int_{\Gamma}(\underline{\underline{\sigma}} \boldsymbol{n}) \cdot \boldsymbol{w}_{s}=-\int_{\Gamma}(p \boldsymbol{n}) \cdot \boldsymbol{w}_{s}
$$

Note that the right hand side of (9) makes sense since $p$ is searched in $H^{1}$. In the same way we use the continuity condition of normal velocities (8a) in the boundary integral of (6a) :

$$
\int_{\Gamma} q\left(\boldsymbol{v}_{f} \cdot \boldsymbol{n}\right)=\int_{\Gamma} q\left(\boldsymbol{v}_{s} \cdot \boldsymbol{n}\right)
$$

Once again the integral of the right hand side of (10) makes sense since $q$ and $\boldsymbol{v}_{s}$ both belong in $H^{1}$. The primal-primal formulation is then :

Find $\left(p, \boldsymbol{v}_{f}, \underline{\underline{\sigma}}, \boldsymbol{v}_{s}\right): t \in \mathbb{R}^{+} \mapsto X$ such that for all $\left(q, \boldsymbol{w}_{f}, \underline{\underline{\xi}}, \boldsymbol{w}_{s}\right)$ in $X$,

$$
(\mathrm{PP})\left\{\begin{array}{l}
\frac{d}{d t} \int_{\Omega_{f}} \frac{p q}{c_{f}^{2} \rho_{f}}-\int_{\Omega_{f}} \boldsymbol{v}_{f} \cdot \nabla q-\int_{\Gamma} q\left(\boldsymbol{v}_{s} \cdot \boldsymbol{n}\right)=0 \\
\frac{d}{d t} \int_{\Omega_{f}} \rho_{s} \boldsymbol{v}_{f} \cdot \boldsymbol{w}_{f}+\int_{\Omega_{f}} \boldsymbol{\nabla} p \cdot \boldsymbol{w}_{f}=0 \\
\frac{d}{d t} \int_{\Omega_{s}} \rho_{s} \boldsymbol{v}_{s} \cdot \boldsymbol{w}_{s}+\int_{\Omega_{s}} \underline{\underline{\sigma}}: \underline{\underline{\nabla}} \boldsymbol{w}_{s}+\int_{\Gamma} p \boldsymbol{n} \cdot \boldsymbol{w}_{s}=0 \\
\frac{d}{d t} \int_{\Omega_{s}} \underline{\underline{\sigma}}: \underline{\underline{\xi}}-\int_{\Omega_{s}} \underline{\underline{C}} \underline{\underline{\nabla}} \boldsymbol{v}_{s}: \underline{\underline{\xi}}=0
\end{array}\right.
$$

where $X=H^{1}\left(\Omega_{f}\right) \times \boldsymbol{L}^{2}\left(\Omega_{f}\right) \times \mathbb{L}^{2}\left(\Omega_{s}\right) \times \boldsymbol{H}^{1}\left(\Omega_{s}\right)$.

The dual-dual formulation We wish here to couple (SD) with (FD). Using the transmission conditions is slightly more delicate than for the primal-primal formulation. Let us 
first explain how we treat the boundary term in (4a).

From the identity $\boldsymbol{u}=(\boldsymbol{u} \cdot \boldsymbol{n}) \boldsymbol{n}+\boldsymbol{n} \wedge(\boldsymbol{u} \wedge \boldsymbol{n})$, the dot product $(\underline{\underline{\xi}} \boldsymbol{n}) \cdot \boldsymbol{v}_{s}$ in the boundary integral of $(4 \mathrm{a})$ is equal to :

$$
((\underline{\underline{\xi}} \boldsymbol{n}) \cdot \boldsymbol{n})\left(\boldsymbol{v}_{s} \cdot \boldsymbol{n}\right)+((\underline{\underline{\xi}}) \wedge \boldsymbol{n}) \cdot\left(\boldsymbol{v}_{s} \wedge \boldsymbol{n}\right)
$$

Let us also notice that the normal stress continuity condition (8b) is equivalent to :

$$
(8 \mathrm{~b}) \quad\left\{\begin{array}{l}
(\underline{\underline{\sigma}} \boldsymbol{n}) \wedge \boldsymbol{n}=0 \\
p=-(\underline{\underline{\sigma}} \boldsymbol{n}) \cdot \boldsymbol{n}
\end{array}\right.
$$

If we strongly impose (12a) in the functional space for tensors, namely :

$$
\underline{\underline{\xi}} \in \mathbb{H}_{\text {div }, 0}^{\text {sym }}\left(\Omega_{s}\right)=\left\{\underline{\underline{\sigma}} \in \mathbb{H}_{\text {div }}^{\text {sym }}\left(\Omega_{s}\right) \mid(\underline{\underline{\sigma}} \boldsymbol{n}) \wedge \boldsymbol{n}=0\right\}
$$

the normal velocity condition (8a) can then be used in the boundary integral (4a) :

$$
\left.\int_{\Gamma} \underline{\underline{\xi}} \boldsymbol{n}\right) \cdot \boldsymbol{v}_{s}=\int_{\Gamma}((\underline{\underline{\xi}} \boldsymbol{n}) \cdot \boldsymbol{n})\left(\boldsymbol{v}_{s} \cdot \boldsymbol{n}\right)=\int_{\Gamma}((\underline{\underline{\xi}} \boldsymbol{n}) \cdot \boldsymbol{n})\left(\boldsymbol{v}_{f} \cdot \boldsymbol{n}\right)
$$

In the same way, for the boundary integral of $(7 \mathrm{~b})$, as soon as $\underline{=}$ is searched in $\mathbb{H}_{\text {div, } 0}^{s y m}\left(\Omega_{s}\right)$, we can use $(12 b)$ to write :

$$
\int_{\Gamma} p\left(\boldsymbol{w}_{f} \cdot \boldsymbol{n}\right)=-\int_{\Gamma}(\underline{\underline{\sigma}} \boldsymbol{n}) \cdot \boldsymbol{n}\left(\boldsymbol{w}_{f} \cdot \boldsymbol{n}\right)
$$

Note that there is still a problem of functional analysis since, if we take $(\underline{\underline{\sigma}}, \underline{\underline{\xi}})$ in $\mathbb{H}_{\text {div, } 0}^{\text {sym }}\left(\Omega_{s}\right)$ and $\left(\boldsymbol{v}_{s}, \boldsymbol{w}_{s}\right)$ in $\boldsymbol{H}_{\text {div }}\left(\Omega_{s}\right)$, the two right hand sides of (13) and (14) appear a priori as integrals of products of functions in $H^{-\frac{1}{2}}(\Gamma)$. That is why we shall look for $\underline{\underline{\sigma}}$ and $\boldsymbol{v}_{s}$ in smaller (and not closed) subspaces, namely :

$$
\left\{\begin{array}{l}
\widehat{\boldsymbol{H}}_{d i v}\left(\Omega_{f}\right)=\left\{\boldsymbol{v} \in \boldsymbol{H}_{\text {div }}\left(\Omega_{f}\right) \mid \boldsymbol{v} \cdot \boldsymbol{n} \in L^{2}(\Gamma)\right\}, \\
\widehat{\mathbb{H}}_{d i v, 0}^{s y m}\left(\Omega_{s}\right)=\left\{\underline{\underline{\sigma}} \in \mathbb{H}_{d i v, 0}^{s y m}\left(\Omega_{s}\right) \mid\left(\underline{\underline{\sigma} \boldsymbol{n})} \cdot \boldsymbol{n} \in L^{2}(\Gamma)\right\},\right.
\end{array}\right.
$$

equipped with their natural Hilbert space norm.

Finally our dual-dual formulation is : 
Find $\left(p, \boldsymbol{v}_{f}, \underline{\underline{\sigma}}, \boldsymbol{v}_{s}\right): t$ in $\mathbb{R}^{+} \mapsto X$ such that for all $\left(q, \boldsymbol{w}_{f}, \underline{\underline{\xi}}, \boldsymbol{w}_{s}\right)$ in $X$,

$$
(\mathrm{DD})\left\{\begin{array}{l}
\frac{d}{d t} \int_{\Omega_{f}} \frac{p q}{c_{f}^{2} \rho_{f}}+\int_{\Omega_{f}} q \operatorname{div} \boldsymbol{v}_{f}=0 \\
\frac{d}{d t} \int_{\Omega_{f}} \rho_{f} \boldsymbol{v}_{f} \cdot \boldsymbol{w}_{f}-\int_{\Omega_{f}} p \operatorname{div} \boldsymbol{w}_{f}+\int_{\Gamma}(\underline{\underline{\sigma}} \boldsymbol{n}) \cdot \boldsymbol{n}\left(\boldsymbol{w}_{f} \cdot \boldsymbol{n}\right)=0 \\
\frac{d}{d t} \int_{\Omega_{s}} \underline{\underline{A}} \underline{\underline{\sigma}}: \underline{\underline{\xi}}+\int_{\Omega_{s}} \operatorname{div} \underline{\underline{\xi}} \cdot \boldsymbol{v}_{s}-\int_{\Gamma}(\underline{\underline{\xi}} \boldsymbol{n}) \cdot \boldsymbol{n}\left(\boldsymbol{v}_{f} \cdot \boldsymbol{n}\right)=0 \\
\frac{d}{d t} \int_{\Omega_{s}} \rho_{s} \boldsymbol{v}_{s} \cdot \boldsymbol{w}_{s}-\int_{\Omega_{s}} \boldsymbol{w}_{s} \cdot \operatorname{div} \underline{\underline{\sigma}}=0
\end{array}\right.
$$

where $X=L^{2}\left(\Omega_{f}\right) \times \widehat{\boldsymbol{H}}_{\text {div }}\left(\Omega_{f}\right) \times \widehat{\mathbb{H}}_{\text {div }, 0}^{\text {sym }}\left(\Omega_{s}\right) \times \boldsymbol{L}^{2}\left(\Omega_{s}\right)$

\section{Space discretization}

We present in this section the finite elements used for the space discretization of the variational formulation. For the dual-dual formulation, we have chosen, in the solid, finite elements constructed from Tsogka's ${ }^{1} Q_{k+1}^{d i v}-Q_{k}$ finite elements. In the fluid, we use the classical Raviart-Thomas' finite elements. For the primal-primal formulation we have chosen Fauqueux's ${ }^{2} Q_{k}$ finites elements in both fluid and solid.

In the following we denote

- $\mathcal{T}_{h_{f}}\left(\right.$ resp. $\mathcal{T}_{h_{s}}$ ) a mesh of $\Omega_{f}$ (resp. $\Omega_{s}$ ) composed of quadrangles (if $d=2$ ) or hexaedra (if $d=3) K_{i}^{f}\left(\right.$ resp. $\left.K_{i}^{s}\right)$;

- $\boldsymbol{F}_{i}^{f}$ (resp. $\boldsymbol{F}_{i}^{s}$ ) : $\widehat{K} \mapsto K_{i}^{f}$ (resp. $K_{i}^{s}$ ) is a one to one vectorial mapping transforming the boundary of $\widehat{K}$ onto the boundary of $K_{i}^{f}\left(\operatorname{resp} . K_{i}^{s}\right)$;

- $\widehat{K}$ is the unit square (or cube) $[0 ; 1]^{d}$.

\subsection{Dual-dual formulation}

In the solid. Tsogka's finite elements are specifically defined for regular meshes, therefore we suppose that all the elements of $\mathcal{T}_{h_{s}}$ are squares. As a consequence, curved interfaces shall be staircase discretized. For a sake of simplicity we restrict ourselves to present the finite element of order $1, Q_{1}^{\text {div }}-Q_{0}$ in dimension 2 and we refer the reader to ${ }^{1}$ for the extension to higher order element or to dimension 3 .

The $Q_{1}^{\text {div }}$ space is the space of stresses which are piecewise $Q_{1}$ and satisfy :

- $\sigma_{11}$ is discontinuous through the horizontal edges of the mesh and continuous through the vertical ones ; 
- $\sigma_{22}$ is discontinuous through the vertical edges of the mesh and continuous through the horizontal ones ;

- $\sigma_{12}=\sigma_{21}$ is a continuous function.

The degrees of freedom of the stresses are all associated to the nodes of the elements (see figure 2). In order to be conforming in the space $\mathbb{H}_{d i v, 0}^{s y m}$ we have to impose that $\sigma_{12}=0$ for all nodes at the fluid-solid interface.

Note that $(\underline{\underline{\sigma} \boldsymbol{n}}) \boldsymbol{n}$ belongs to $L^{2}(\Gamma)$ for all $\underline{\underline{\sigma}}$ in $Q_{1}^{\text {div }}$.

The $Q_{0}$ space is the space of piecewise constant velocity fields (the degrees of freedom are located at the center of the element - see figure 2).

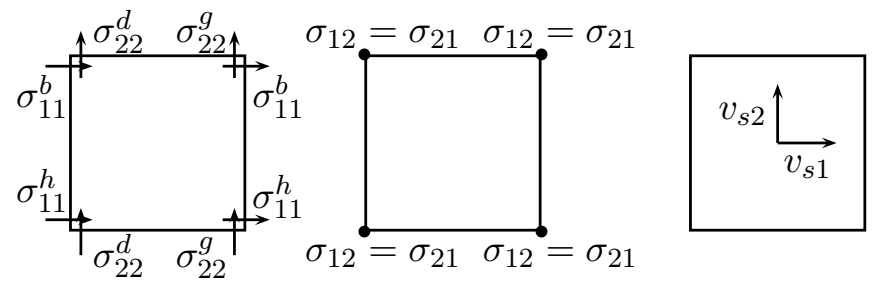

Fig. 2. Finite element in the solid : the stresses (left and center) and the velocities(right)

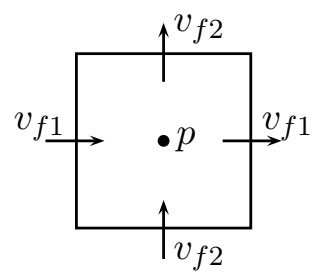

Fig. 3. Finite element used in the fluid

In the fluid. The pressure is piecewise constant (it belongs to $Q_{0}$ ) and Raviart-Thomas's finite elements are used for the velocities (see figure 3).

Semi-discretized equations. Applying the standard finite element procedure leads to the following ordinary differential system where the vectors $P, V_{f}, V_{s}$ and $\Sigma$ are composed of the degrees of freedom of $p, v_{f}, v_{s}$ and $\sigma$ :

$$
\left\{\begin{array}{l}
M_{P} \frac{d P}{d t}+D_{f} V_{f}=0 \\
M_{f} \frac{d V_{f}}{d t}-D_{f}^{*} P-B \Sigma,=0 \\
M_{\Sigma} \frac{d \Sigma}{d t}+D_{s}^{*} V_{s}+B^{*} V_{f}=0 \\
M_{s} \frac{d V_{s}}{d t}-D_{s} \Sigma=0
\end{array}\right.
$$


The matrices $D_{f}$ and $D_{s}$ represent discrete divergence operators and $B$ and $B^{*}$ discrete trace operators. Since $p$ and $v_{s}$ are piecewise constant, the matrices $M_{P}$ and $M_{s}$ are diagonal. We apply the mass-lumping technique describe in $^{3}$ in order to obtain the matrices $M_{f}$ diagonal and $M_{\Sigma}(5 \times 5)$ block-diagonal.

\subsection{Primal-primal formulation}

In view of using Fauqueux's finite element in the solid, we have to modify the formulation (PP) by introducing an auxiliary function $\frac{\partial \underline{\underline{\underline{\gamma}}}}{\partial t}=\underline{\underline{\nabla}} \boldsymbol{v}_{s}$. The formulation, equivalent to (PP), that will be used for the discretization is the following :

Find $\left(\left(p, \boldsymbol{v}_{f}, \underline{\underline{\sigma}}, \boldsymbol{v}_{s}\right), \gamma\right): t \in \mathbb{R}^{+} \mapsto X \times \mathbb{L}^{2}\left(\Omega_{s}\right)$ such that for all $\left(q, \boldsymbol{w}_{f}, \underline{\underline{\xi}}, \boldsymbol{w}_{s}\right)$ in $X$,

$$
\left(\mathrm{PP}^{\prime}\right)\left\{\begin{array}{l}
\frac{d}{d t} \int_{\Omega_{f}} \frac{p q}{c_{f}^{2} \rho_{f}}-\int_{\Omega_{f}} \boldsymbol{v}_{f} \cdot \nabla q-\int_{\Gamma} q\left(\boldsymbol{v}_{s} \cdot \boldsymbol{n}\right)=0 \\
\frac{d}{d t} \int_{\Omega_{f}} \rho_{f} \boldsymbol{v}_{f} \cdot \boldsymbol{w}_{f}+\int_{\Omega_{f}} \boldsymbol{\nabla} p \cdot \boldsymbol{w}_{f}=0 \\
\frac{d}{d t} \int_{\Omega_{s}} \rho_{s} \boldsymbol{v}_{s} \cdot \boldsymbol{w}_{s}+\int_{\Omega_{s}} \underline{\underline{\sigma}}: \underline{\underline{\nabla}} \boldsymbol{w}_{s}+\int_{\Gamma} p \boldsymbol{n} \cdot \boldsymbol{w}_{s}=0 \\
\int_{\Omega_{s}} \underline{\underline{\sigma}}: \underline{\underline{\xi}}-\int_{\Omega_{s}}^{\underline{C}} \underline{\underline{\gamma}}: \underline{\underline{\xi}}=0 \\
\frac{d}{d t} \int_{\Omega_{s}} \underline{\underline{\gamma}}: \underline{\underline{\xi}}-\int_{\Omega_{s}} \underline{\underline{\nabla}}=\underline{\underline{\underline{\xi}}}=0
\end{array}\right.
$$

where $X=H^{1}\left(\Omega_{f}\right) \times \boldsymbol{L}^{2}\left(\Omega_{f}\right) \times \mathbb{L}^{2}\left(\Omega_{s}\right) \times \boldsymbol{H}^{1}\left(\Omega_{s}\right)$.

The $H^{1}$ functions $p$ and $\boldsymbol{v}_{s}$ are approximated by continuous functions whose respective restrictions $p^{i}$ and $\boldsymbol{v}_{s}^{j}$ to $K_{i}^{f}$ and $K_{j}^{s}$ are such that $\widehat{p}^{i}=p^{i} \circ \boldsymbol{F}_{i}^{f}$ and $\widehat{\boldsymbol{v}}_{s}^{j}=\boldsymbol{v}_{s}^{j} \circ \boldsymbol{F}_{j}^{s}$ are $Q_{k}$ in $\widehat{K}$. Moreover, in view of mass lumping, the degrees of freedom associated to $p^{i}$ and $\boldsymbol{v}_{s}^{j}$ are chosen to coincide with the $Q_{k}$ Gauss-Lobatto quadrature points.

The fields $\boldsymbol{v}_{f}$ and $\underline{\underline{\sigma}}$ are approximated by discontinuous fields whose respective restrictions $\boldsymbol{v}_{f}^{i}$ and $\underline{\underline{\sigma}}^{j}$ to $K_{i}^{f}$ and $K_{j}^{s}$ are such that (this is the Piola transform, $J_{i}^{l}$ is the Jacobian of $\left.\boldsymbol{F}_{i}^{l}, l=\bar{f}, s\right)$ :

$$
\widehat{\boldsymbol{v}}_{f}^{i}=\left|J_{i}^{f}\right| D \boldsymbol{F}_{i}^{f-1} \boldsymbol{v}_{f}^{i} \circ \boldsymbol{F}_{i}^{f} \quad \text { and } \quad{\underline{\underline{\sigma^{\prime}}}}^{j}=\left|J_{j}^{s}\right| D \boldsymbol{F}_{j}^{s-1} \underline{\underline{\sigma}}^{j} \circ \boldsymbol{F}_{j}^{s} \quad \text { are } Q_{k} \text { in } \widehat{K}
$$


With obvious notation we end up with the following ordinary differential system ;

$$
\left\{\begin{array}{l}
M_{P} \frac{d P}{d t}-G_{f}^{*} V_{f}-B V_{s}=0 \\
M_{f} \frac{d V_{f}}{d t}+G_{f} P=0 \\
M_{s} \frac{d V_{s}}{d t}+G_{s}^{*} \Sigma+B^{*} P=0 \\
M_{\Sigma} \Sigma-C \Gamma=0 \\
M_{\Sigma} \frac{d \Gamma}{d t}-G_{s} V_{s}=0
\end{array}\right.
$$

where the matrices $G_{f}$ and $G_{s}$ are discrete gradient operators and $C$ approximates the multiplication by tensor $\underline{\underline{C}}$. The mass lumping procedure is once again applied, so that the matrices $M_{P}$, and $M_{s}$ are diagonal, $M_{f}$ and $M_{\Sigma}$ are $(d \times d)$ block-diagonal and $\boldsymbol{C}$ is $\left(d^{2} \times d^{2}\right)$ block-diagonal.

Remark 4.1. In the practical computation, the unknown $\Gamma$ is eliminated and the two equations (19d) and (19e) are replaced by

$$
M_{\Sigma} \frac{d \Sigma}{d t}-C M_{\Sigma}^{-1} G_{s} V_{s}=0
$$

Remark 4.2. Let us recall that an interesting property of the choice (18) is

$$
\int_{K_{i}^{f}} \boldsymbol{\nabla} p^{i} \cdot \boldsymbol{v}_{f}^{j}=\int_{\widehat{K}} \nabla \widehat{p}^{i} \cdot \widehat{\boldsymbol{v}}_{f}^{j} \quad \text { and } \quad \int_{K_{i}^{s}} \underline{\underline{\nabla}} \boldsymbol{v}_{s}^{i}: \underline{\underline{\sigma}}^{j}=\int_{\widehat{K}} \underline{\underline{\nabla}} \widehat{\boldsymbol{v}}_{s}^{i}: \underline{\underline{\widehat{\sigma}}}^{j} .
$$

As a consequence, the matrices $G_{f}$ and $G_{s}$ are (quasi) independent on the meshes $\mathcal{T}_{h_{f}}$ and $\mathcal{T}_{h_{s}}$ (only their topology is involved) : all the information about the coefficients and the geometry of the meshes is contained in the diagonal matrices $M_{P}, M_{f}, M_{s}, M_{\Sigma}$ and $\boldsymbol{C}$.

\section{Time discretization}

We now concentrate ourselves on the semi-discretized scheme obtained from the primalprimal formulation, but the following can also be applied to the dual-dual formulation.

We intend to obtain second order time-centered schemes. The most natural way to do so is to compute $P$ et $V_{f}$ at shifted instants ( $n$ and $n+\frac{1}{2}$ for instance - see figure 4 ), so that (19a) and (19b) could be explicitly solved when the coupling terms cancel out. We proceed in the same way for $\Sigma$ and $V_{s}$ and compute (19c) and (19d) explicitly (see figure 5). 


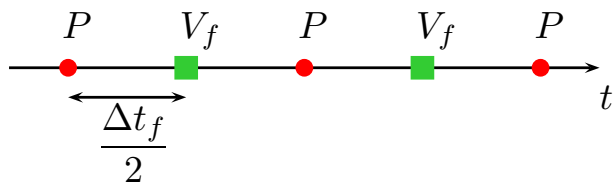

Fig. 4. Time discretization in the fluid

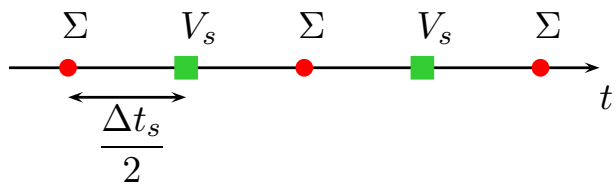

Fig. 5. Time discretization in the solid

We will first present the time-discretization in the case of a conforming scheme $\left(\Delta t_{f}=\Delta t_{s}\right)$ before considering a particular non-conforming case (namely $\Delta t_{f}=2 \Delta t_{s}$ ).

\subsection{Using the same time step in both domains}

Two choices are possible :

(1) compute $V_{s}$ and $P$ at shifted instants (see figure 6) and obtain a completely explicit scheme ;

(2) compute $V_{s}$ and $P$ at the same instants (see figure 7 ), which leads to an explicit scheme for the internal nodes but not on the interface.

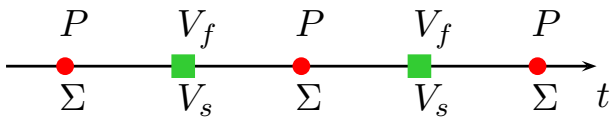

Fig. 6. Time discretization, choice (1)

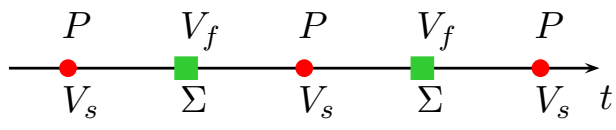

Fig. 7. Time discretization, choice (2)

Fully explicit scheme. We consider choice (1) and apply the following scheme :

$$
\left\{\begin{array}{l}
M_{P} \frac{P^{n+1}-P^{n}}{\Delta t}-G_{f}^{*} V_{f}^{n+\frac{1}{2}}-B V_{s}^{n+\frac{1}{2}}=0 \\
M_{f} \frac{V_{f}^{n+\frac{1}{2}}-V_{f}^{n-\frac{1}{2}}}{\Delta t}+G_{f} P^{n}=0 \\
M_{s} \frac{V_{s}^{n+\frac{1}{2}}-V_{s}^{n-\frac{1}{2}}}{\Delta t}+G_{s}^{*} \Sigma^{n}+B^{*} P^{n}=0 \\
M_{\Sigma} \frac{\Sigma^{n+1}-\Sigma^{n}}{\Delta t}-C M_{\Sigma}^{-1} G_{s} V_{s}^{n+\frac{1}{2}}=0
\end{array}\right.
$$

Since only the inversion of the mass matrices is involved, the scheme is fully explicit. The disadvantage is that the stability condition of the scheme seems to be affected by the transmission conditions (it does not coincide with the stability condition imposed in the two interior schemes - see below). 
Semi-explicit scheme. We consider choice (2) and apply the following centered discretization procedure :

$$
\left\{\begin{array}{l}
M_{P} \frac{P^{n+\frac{1}{2}}-P^{n-\frac{1}{2}}}{\Delta t}-G_{f}^{*} V_{f}^{n}-B \frac{V_{s}^{n+\frac{1}{2}}+V_{s}^{n-\frac{1}{2}}}{2}=0 \\
M_{f} \frac{V_{f}^{n+1}-V_{f}^{n}}{\Delta t}+G_{f} P^{n+\frac{1}{2}}=0 \\
M_{s} \frac{V_{s}^{n+\frac{1}{2}}-V_{s}^{n-\frac{1}{2}}}{\Delta t}+G_{s}^{*} \Sigma^{n}+B^{*} \frac{P^{n+\frac{1}{2}}+P^{n-\frac{1}{2}}}{2}=0 \\
M_{\Sigma} \frac{\Sigma^{n+1}-\Sigma^{n}}{\Delta t}-C M_{\Sigma}^{-1} G_{s} V_{s}^{n+\frac{1}{2}}=0
\end{array}\right.
$$

It is obvious from equations $(22 \mathrm{~b})$ and $(22 \mathrm{~d})$ that $V_{f}$ and $\Sigma$ can be computed explicitly, while $P$ and $V_{s}$ are obtained by solving a system of the form :

$$
\left\{\begin{array}{l}
M_{P} P^{n+\frac{1}{2}}-\frac{\Delta t}{2} B V_{s}^{n+\frac{1}{2}}=F^{n+\frac{1}{2}} \\
M_{s} V_{s}^{n+\frac{1}{2}}+\frac{\Delta t}{2} B^{*} P^{n+\frac{1}{2}}=G^{n+\frac{1}{2}}
\end{array}\right.
$$

where $F^{n+\frac{1}{2}}$ and $G^{n+\frac{1}{2}}$ are known from previous time steps. The invertibilty of (23a) and (23b) is guaranteed by the fact that

$$
M_{P}+\frac{\Delta t^{2}}{4} B M_{s}^{-1} B^{*}
$$

is symmetric positive definite. Moreover, as the matrices $M_{P}$ and $M_{s}$ are both diagonal and as the matrix $B$ only "sees" the interface, the scheme is implicit only for the degrees of freedom of $P$ and $V_{s}$ that are located on the interface. The "boundary matrix" to be inverted is accordingly very sparse.

On the stability analysis of schemes I and II. The stability of both schemes can be analyzed by energy techniques. If $n$ is an integer, we introduce the two discrete energies :

$$
E_{f}^{n+\frac{1}{2}}=\frac{1}{2}\left[\left(M_{P} P^{n+\frac{1}{2}}, P^{n+\frac{1}{2}}\right)+\left(M_{f} V_{f}^{n+1}, V_{f}^{n}\right)\right]
$$

and

$$
E_{s}^{n+\frac{1}{2}}=\frac{1}{2}\left[\left(M_{s} V_{s}^{n+\frac{1}{2}}, V_{s}^{n+\frac{1}{2}}\right)+\left(C \Gamma^{n+1}, \Gamma^{n}\right)\right],
$$

where the sequence $\Gamma^{n}$ is defined by the following equation :

$$
M_{\Sigma} \frac{\Gamma^{n+1}-\Gamma^{n}}{\Delta t}-G_{s} V_{s}^{n+\frac{1}{2}}=0
$$


so that

$$
M_{\Sigma} \Sigma^{n}=C \Gamma^{n}
$$

$E_{f}^{n+\frac{1}{2}}$ and $E_{s}^{n+\frac{1}{2}}$ are respectively discrete equivalent of the continuous energies

$$
E_{f}=\int_{\Omega_{f}} \frac{p^{2}}{c_{f}^{2} \rho_{f}}+\int_{\Omega_{f}} \rho_{f}\left|\boldsymbol{v}_{f}\right|^{2}
$$

and

$$
E_{s}=\int_{\Omega_{s}} \rho_{s}\left|\boldsymbol{v}_{s}\right|^{2}+\int_{\Omega_{s}} \underline{\underline{C}} \underline{\underline{\underline{\sigma}}}: \underline{\underline{\sigma}}=\int_{\Omega_{s}} \rho_{s}\left|\boldsymbol{v}_{s}\right|^{2}+\int_{\Omega_{s}} \underline{\underline{C}} \underline{\underline{\gamma}}: \underline{\underline{\gamma}}
$$

Each of this discrete energies would be conserved in the absence of coupling. With the coupling scheme II (respectively I) one can show the conservation of the global discrete energy :

$$
E_{I I}^{n+\frac{1}{2}}=E_{f}^{n+\frac{1}{2}}+E_{s}^{n+\frac{1}{2}}, \quad\left(\operatorname{respectively} E_{I}^{n+\frac{3}{4}}=E_{f}^{n+1}+E_{s}^{n+\frac{1}{2}}-\Delta t\left(B V_{s}^{n+\frac{1}{2}}, P^{n+1}\right)\right) .
$$

Therefore, the $L^{2}$ stability of the scheme follows from the positivity of each energy.

For scheme II, it is clear that the positivity of $E_{I I}^{n+\frac{1}{2}}$ amounts to the one of $E_{f}^{n+\frac{1}{2}}$ and $E_{s}^{n+\frac{1}{2}}$.

For the positivity of $E_{f}^{n+\frac{1}{2}}$, we rewrite the scalar product $\left(M_{f} V_{f}^{n+1}, V_{f}^{n}\right)$ as

$$
\left(M_{f} \frac{V_{f}^{n+1}+V_{f}^{n}}{2}, \frac{V_{f}^{n+1}+V_{f}^{n}}{2}\right)-\frac{\Delta t^{2}}{4}\left(M_{f} \frac{V_{f}^{n+1}-V_{f}^{n}}{\Delta t}, \frac{V_{f}^{n+1}-V_{f}^{n}}{\Delta t}\right) .
$$

Then equation (22b) leads to

$$
\left(M_{f} \frac{V_{f}^{n+1}-V_{f}^{n}}{\Delta t}, \frac{V_{f}^{n+1}-V_{f}^{n}}{\Delta t}\right)=\left(G_{f}^{*} M_{f}^{-1} G_{f} P^{n+\frac{1}{2}}, P^{n+\frac{1}{2}}\right)
$$

and $E_{f}^{n+\frac{1}{2}}$ becomes

$$
E_{f}^{n+\frac{1}{2}}=\frac{1}{2}\left[\left(M_{f} \frac{V_{f}^{n+1}+V_{f}^{n}}{2}, \frac{V_{f}^{n+1}+V_{f}^{n}}{2}\right)+\left(\left[M_{P}-\frac{\Delta t^{2}}{4} G_{f}^{*} M_{f}^{-1} G_{f}\right] P^{n+\frac{1}{2}}, P^{n+\frac{1}{2}}\right)\right] .
$$

Using the same way it is clear that :

$E_{s}^{n+\frac{1}{2}}=\frac{1}{2}\left[\left(\boldsymbol{C} \frac{\Gamma^{n+1}+\Gamma^{n}}{2}, \frac{\Gamma^{n+1}+\Gamma^{n}}{2}\right)+\left(\left[M_{s}-\frac{\Delta t^{2}}{4} G_{s}^{*} M_{\Sigma}^{-1} \boldsymbol{C} M_{\Sigma}^{-1} G_{s}\right] V_{s}^{n+\frac{1}{2}}, V_{s}^{n+\frac{1}{2}}\right)\right]$.

Thus $E_{I I}^{n+\frac{1}{2}}$ will be positive if the matrices

$$
M_{P}-\frac{\Delta t^{2}}{4} G_{f}^{*} M_{f}^{-1} G_{f} \quad \text { and } \quad M_{s}-\frac{\Delta t^{2}}{4} G_{s}^{*} M_{\Sigma}^{-1} C M_{\Sigma}^{-1} G_{s}
$$


are definite positive. It has been shown ${ }^{2}$ that the first one was so if

$$
\Delta t \leq \alpha_{r} \frac{h_{f}}{c_{f} \sqrt{d}}
$$

and that the second one was so if

$$
\Delta t \leq \alpha_{r} \frac{h_{s}}{c_{P} \sqrt{d}}
$$

where $\alpha_{r}$ depends on the order of the finite element method, as described $\mathrm{in}^{2}$, for instance $\alpha_{5} \simeq 0.101$.

Finally the scheme II is stable (i.e. the energy $E^{\frac{1}{2}}$ is positive) if the following stability condition is satisfied :

$$
\Delta t \leq \alpha_{r} \min \left(\frac{h_{s}}{c_{P} \sqrt{d}}, \frac{h_{f}}{c_{f} \sqrt{d}}\right) .
$$

For scheme I it is clear that condition $(28)$ is a necessary condition for the positivity of $E_{I}$ (consider separately $V_{s}^{n+\frac{1}{2}}=0$ and $P^{n+1}=0$ ). However, one also has to ensure that the additional term

$$
\Delta t\left(B V_{s}^{n+\frac{1}{2}}, P^{n+1}\right)
$$

can not be too large, which provides an additional condition on the matrix $\Delta t B$ that must not be too large. This leads to an additional CFL-like condition of the type

$$
\Delta t \leq \alpha_{c} \frac{h_{s}}{c_{P} \sqrt{d}}
$$

with $\alpha_{c} \leq \alpha_{r}$. In this sense the stability condition of scheme I is affected by the coupling procedure and that is why, for robustness purpose the scheme II will be preferred.

Remark 5.1. The numerical experiments we have achieved seem to show that $\alpha_{c}$ is close to $\alpha_{r}$ if one uses the primal-primal method whereas it could be much less than $\alpha_{r}$ if one uses the dual-dual method.

\subsection{Local time-stepping}

We present here an adaptation of the multiplier free mesh refinement technique ${ }^{3,6}$ to the fluid-structure coupling problem. We describe here the case where $\Delta t_{f}=2 \Delta t_{s}=2 \Delta t$ (scheme 1-2), but this could be extended to any rational ratio $p-q$ by adaptating the results presented $\mathrm{in}^{6}$. Here again several schemes could be consider. We shall present a scheme that possesses the same characteristics and the same robustness properties that the scheme II presented above :

- the scheme is explicit in the interior domain and implicit on the interface ;

- the scheme conserves some discrete energy and the stability condition is not affected by the coupling procedure. 
We choose to compute the unknown $P$ at time $t^{2 n}=2 n \Delta t, V_{f}$ at time $t^{2 n+1}, V_{s}$ at time $t^{n}$ and $\Sigma$ at time $t^{n+\frac{1}{2}}$ (see figure 8 ). In the time interval $\left[t^{2 n} ; t^{2 n+2}\right]$ we shall use the following system :

- in the fluid :

$$
\left\{\begin{array}{l}
M_{P} \frac{P^{2 n+2}-P^{2 n}}{2 \Delta t}-G_{f}^{*} V_{f}^{2 n+1}-B\left[V_{s}\right]^{2 n+1}=0 \\
M_{f} \frac{V_{f}^{2 n+1}-V_{f}^{2 n-1}}{2 \Delta t}+G_{f} P^{2 n}=0
\end{array}\right.
$$

- in the solid (first time step) :

$$
\text { (III.s1) }\left\{\begin{array}{l}
M_{s} \frac{V_{s}^{2 n+1}-V_{s}^{2 n}}{\Delta t}+G_{s}^{*} \Sigma^{2 n+\frac{1}{2}}+B^{*}[P]^{2 n+\frac{1}{2}}=0, \\
M_{\Sigma} \frac{\Sigma^{2 n+\frac{1}{2}}-\Sigma^{2 n-\frac{1}{2}}}{\Delta t}-C M_{\Sigma}^{-1} G_{s} V_{s}^{2 n}=0
\end{array}\right.
$$

- in the solid (second time step) :

$$
\left(\text { III.s2) } \left\{\begin{array}{l}
M_{s} \frac{V_{s}^{2 n+2}-V_{s}^{2 n+1}}{\Delta t}+G_{s}^{*} \Sigma^{2 n+\frac{3}{2}}+B^{*}[P]^{2 n+\frac{3}{2}}=0 \\
M_{\Sigma} \frac{\Sigma^{2 n+\frac{3}{2}}-\Sigma^{2 n+\frac{1}{2}}}{\Delta t}-C M_{\Sigma}^{-1} G_{s} V_{s}^{2 n+1}=0
\end{array}\right.\right.
$$

where $\left[V_{s}\right]^{2 n+1}$, (respectively $[P]^{2 n+\frac{1}{2}}$ and $\left.[P]^{2 n+\frac{3}{2}}\right)$ represents some approximations of $V_{s}$ at time $2 n+1$ (respectively $P$ at time $2 n+\frac{1}{2}$ and at time $2 n+\frac{3}{2}$ ) that remain to be define. This terms are determined to get an energy conservation result.

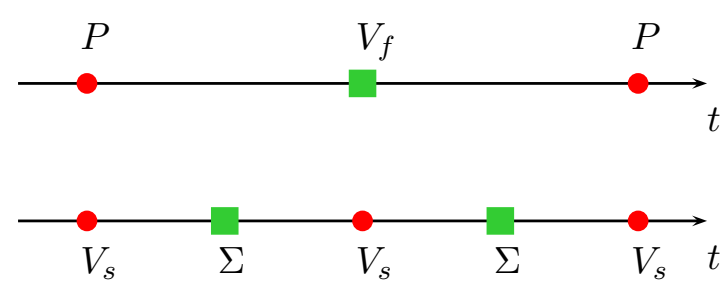

Fig. 8. Time discretization, scheme III

An energy can be deduced in the fluid :

$$
E_{f}^{2 n}=\frac{1}{2}\left[\left(M_{P} P^{2 n}, P^{2 n}\right)+\left(M_{f} V_{f}^{2 n+1}, V_{f}^{2 n-1}\right)\right],
$$


and in the solid :

$$
E_{s}^{2 n}=\frac{1}{2}\left[\left(M_{s} V_{s}^{2 n}, V_{s}^{2 n}\right)+\left(C \Gamma^{2 n+\frac{1}{2}}, \Gamma^{2 n-\frac{1}{2}}\right)\right]
$$

where the sequence $\Gamma^{n+\frac{1}{2}}$ is defined by the following equation :

$$
M_{\Sigma} \frac{\Gamma^{n+\frac{1}{2}}-\Gamma^{n}}{\Delta t}-G_{s} V_{s}^{n}=0
$$

Denoting $E^{2 n}=E_{f}^{2 n}+E_{s}^{2 n}$ (that is obviously a positive energy under the condition (28)), it can easily be shown that :

$$
\begin{aligned}
\frac{E^{2 n+2}-E^{2 n}}{2 \Delta t} & =B^{*}[P]^{2 n+\frac{3}{2}} \frac{V_{s}^{2 n+2}+V_{s}^{2 n+1}}{4}+B^{*}[P]^{2 n+\frac{1}{2}} \frac{V_{s}^{2 n+1}+V_{s}^{2 n}}{4} \\
& -B\left[V_{s}\right]^{2 n+1}\left(\frac{P^{2 n+2}+P^{2 n}}{2}\right)
\end{aligned}
$$

As a consequence a conservative scheme is obtained by choosing :

$$
[P]^{2 n+\frac{1}{2}}=[P]^{2 n+\frac{3}{2}}=\frac{P^{2 n+2}+P^{2 n}}{2}
$$

and

$$
\left[V_{s}\right]^{2 n+1}=\frac{V_{s}^{2 n+2}+2 V_{s}^{2 n+1}+V_{s}^{2 n}}{4} .
$$

The system (30) then becomes:

- in the fluid :

$$
\text { (III.f) }\left\{\begin{array}{l}
M_{P} \frac{P^{2 n+2}-P^{2 n}}{2 \Delta t}-G_{f}^{*} V_{f}^{2 n+1}-B \frac{V_{s}^{2 n+2}+2 V_{s}^{2 n+1}+V_{s}^{2 n}}{4}=0 \\
M_{f} \frac{V_{f}^{2 n+1}-V_{f}^{2 n-1}}{2 \Delta t}+G_{f} P^{2 n}=0
\end{array}\right.
$$

- in the solid (first time step) :

$$
\text { (III.s1) }\left\{\begin{array}{l}
M_{s} \frac{V_{s}^{2 n+1}-V_{s}^{2 n}}{\Delta t}+G_{s}^{*} \Sigma^{2 n+\frac{1}{2}}+B^{*} \frac{P^{2 n+2}+P^{2 n}}{2}=0 \\
M_{\Sigma} \frac{\Sigma^{2 n+\frac{1}{2}}-\Sigma^{2 n-\frac{1}{2}}}{\Delta t}-C M_{\Sigma}^{-1} G_{s} V_{s}^{2 n}=0
\end{array}\right.
$$


- in the solid (second time step) :

$$
\text { (III.s2) }\left\{\begin{array}{l}
M_{s} \frac{V_{s}^{2 n+2}-V_{s}^{2 n+1}}{\Delta t}+G_{s}^{*} \Sigma^{2 n+\frac{3}{2}}+B^{*} \frac{P^{2 n+2}+P^{2 n}}{2}=0 \\
M_{\Sigma} \frac{\Sigma^{2 n+\frac{3}{2}}-\Sigma^{2 n+\frac{1}{2}}}{\Delta t}-C M_{\Sigma}^{-1} G_{s} V_{s}^{2 n+1}=0 .
\end{array}\right.
$$

Existence and uniqueness of the solution of (34). The unknowns

$$
V_{f}^{2 n+1} \text { and } \Sigma^{2 n+\frac{1}{2}}
$$

can be computed explicitly thanks to equations (34b) and (34d). The following system remains to be solved :

$$
\left\{\begin{array}{l}
M_{P} P^{2 n+2}-2 \Delta t B \frac{V_{s}^{2 n+2}+2 V_{s}^{2 n+1}}{4}=F_{1}^{n} \\
M_{s} V_{s}^{2 n+1}+\Delta t B^{*} \frac{P^{2 n+2}}{2}=F_{2}^{n} \\
M_{s}\left[V_{s}^{2 n+2}-V_{s}^{2 n+1}\right]+\Delta t G_{s}^{*} \Sigma^{2 n+\frac{3}{2}}+\Delta t B^{*} \frac{P^{2 n+2}}{2}=F_{3}^{n} \\
M_{\Sigma} \Sigma^{2 n+\frac{3}{2}}-\Delta t C M_{\Sigma}^{-1} G_{s} V_{s}^{2 n+1}=F_{4}^{n},
\end{array}\right.
$$

where the vectors $F_{i}^{n}$ can be deduced from the value of the unknowns at time $2 n$. It is clear that $V_{s}^{2 n+1}, \Sigma^{2 n+\frac{3}{2}}$ and $V_{s}^{2 n+2}$ can be explicitly computed as soon as $P^{2 n+2}$ is known, while it can be proved, by some calculation, that this latter is solution of

$$
\left[M_{P}+\Delta t^{2} B M_{s}^{-1}\left[M_{s}-\frac{\Delta t^{2}}{4} G_{s}^{*}\left[M_{\Sigma}^{-1} C M_{\Sigma}^{-1}\right] G_{s}\right] M_{s}^{-1} B^{*}\right] P^{2 n+2}=F_{5}^{n}
$$

where the vector $F_{5}^{n}$ can be deduced from the value of the unknowns at time $2 n$. The reader will easily realize that one is reduce in practice to the resolution of a sparse positive definite symmetric system for the boundary component of $P^{2 n+2}$ (remember that $B$ represent some discrete trace operator). Indeed we know from section 5.1 that the matrix

$$
A=M_{s}-\frac{\Delta t^{2}}{4} G_{s}^{*}\left[M_{\Sigma}^{-1} C M_{\Sigma}^{-1}\right] G_{s}
$$

is symmetric positive definite as soon as the CFL condition (26) is satisfied, thus so is

$$
M_{P}+\Delta t^{2} B M_{s}^{-1} A M_{s}^{-1} B^{*}
$$

and equation (36) admits one unique solution. 
A post-treated scheme. Clearly, the transmission conditions (32) are only first order accurate. A consequence of that is the appearance of parasitic evanescent waves confined at the interface. To overcome this difficulty, in ${ }^{6}$ the following post-treatment is proposed.

- the standard mean of the unknowns over two time steps in the fluid :

$$
\boldsymbol{P}^{2 n+1}=\frac{P^{n+2}+P^{n}}{2} \text { and } \boldsymbol{V}_{f}^{2 n}=\frac{V_{f}^{2 n+1}+V_{f}^{2 n-1}}{2}
$$

- the following weighted mean of the unknowns over three time steps in the solid :

$$
\boldsymbol{V}_{s}^{2 n}=\frac{V_{s}^{2 n+1}+2 V_{s}^{2 n}+V_{s}^{2 n-1}}{4} \text { and } \Sigma^{2 n+\frac{1}{2}}=\frac{\Sigma^{2 n+\frac{3}{2}}+2 \Sigma^{2 n+\frac{1}{2}}+\Sigma^{2 n-\frac{1}{2}}}{4}
$$

Then scheme III can also be reinterpreted as follows :

- in the fluid :

$$
\left(\text { III'.f) } \left\{\begin{array}{l}
M_{P} \frac{\boldsymbol{P}^{2 n+1}-\boldsymbol{P}^{2 n-1}}{2 \Delta t}-G_{f}^{*} \boldsymbol{V}_{f}^{2 n}-B \frac{\boldsymbol{V}_{s}^{2 n+1}+\boldsymbol{V}_{s}^{2 n-1}}{2}=F^{2 n}, \\
M_{f} \frac{\boldsymbol{V}_{f}^{2 n}-\boldsymbol{V}_{f}^{2 n-2}}{2 \Delta t}+G_{f} \boldsymbol{P}^{2 n-1}=0,
\end{array}\right.\right.
$$

- in the solid (first time step) :

$$
\text { (III'.s1) }\left\{\begin{array}{l}
M_{s} \frac{\boldsymbol{V}_{s}^{2 n}-\boldsymbol{V}_{s}^{2 n+1}}{\Delta t}+G_{s}^{*} \Sigma^{2 n-\frac{1}{2}}+B^{*} \frac{\boldsymbol{P}^{2 n+1}+3 \boldsymbol{P}^{2 n-1}}{4}=0, \\
M_{\Sigma} \frac{\boldsymbol{\Sigma}^{2 n-\frac{1}{2}}-\boldsymbol{\Sigma}^{2 n-\frac{3}{2}}}{\Delta t}-\boldsymbol{C} M_{\Sigma}^{-1} G_{s} \boldsymbol{V}_{s}^{2 n-1}=0,
\end{array}\right.
$$

- in the solid (second time step) :

$$
\text { (III'.s2) }\left\{\begin{array}{l}
M_{s} \frac{\boldsymbol{V}_{s}^{2 n+1}-\boldsymbol{V}_{s}^{2 n}}{\Delta t}+G_{s}^{*} \boldsymbol{\Sigma}^{2 n+\frac{1}{2}}+B^{*} \frac{3 \boldsymbol{P}^{2 n+1}+\boldsymbol{P}^{2 n-1}}{4}=0, \\
M_{\Sigma} \frac{\boldsymbol{\Sigma}^{2 n+\frac{1}{2}}-\boldsymbol{\Sigma}^{2 n-\frac{1}{2}}}{\Delta t}-\boldsymbol{C} M_{\Sigma}^{-1} G_{s} \boldsymbol{V}_{s}^{2 n}=0 .
\end{array}\right.
$$

Remark 5.2. The new scheme has the same stability property as scheme III, however we remark that the coupling terms are this time second order accurate, that results into a gain of accuracy.

\section{Numerical Results}

In this last section we present various numerical results that first aim at showing that the methods proposed in this paper provide accurate numerical solutions (via the comparison with exact solutions), in particular for interface phenomena (section 6.1). Our next results 
aim at proving that our methods are able to handle efficiently complex problems such as the propagation of ultra-sounds waves in the bone (section 6.2).

\subsection{Comparison with analytical solutions}

In two dimensions. In order to validate both the primal-primal and the dual-dual code in two dimensions, we have compared the solution obtained with both methods to an analytical one obtained by the Cagniard-de Hoop technique ${ }^{5,4}$ in a simple configuration : two half-planes separated by a straight interface, one constitutes the fluid medium and the second one constitutes the solid medium. Since this is not the main purpose of this article, we shall not give here neither the details of the calculation of this analytical solution (which are rather technical), nor the expression of this solution (which is rather complicated and has no particular interest in the context of this paper). Let us simply mention that the Cagniardde Hoop method provides a closed form explicit expression of the Green's function of the problem associated to a point source in the fluid. Then, a general solution can be obtained by a simple convolution with the source term that can be computed with a Matlab code with an arbitrary accuracy. In the experiments we consider, the physical parameters for the fluid are $c_{f}=1500 \mathrm{~m} \mathrm{~s}^{-1}$ and $\rho_{f}=1000 \mathrm{~kg} \mathrm{~m}^{-3}$ and the physical parameters for the solid are $c_{S}=1800 \mathrm{~m} \mathrm{~s}^{-1}, c_{P}=4000 \mathrm{~m} \mathrm{~s}^{-1}$ and $\rho_{s}=1850 \mathrm{~kg} \mathrm{~m}^{-3}$. For the numerical computation the size of the computational domain in each medium is $20 \mathrm{~mm} \times 5 \mathrm{~mm}$ and Perfectly Matched Layers are used to simulate infinite half spaces. The source function is a point source in space, (located in the fluid at $2 \mathrm{~mm}$ from the interface - see figure 9 ) whose time variation is given as the first derivative of a Gaussian whose dominating frequency is $1 \mathrm{MHz}$.

We compare two numerical calculations :

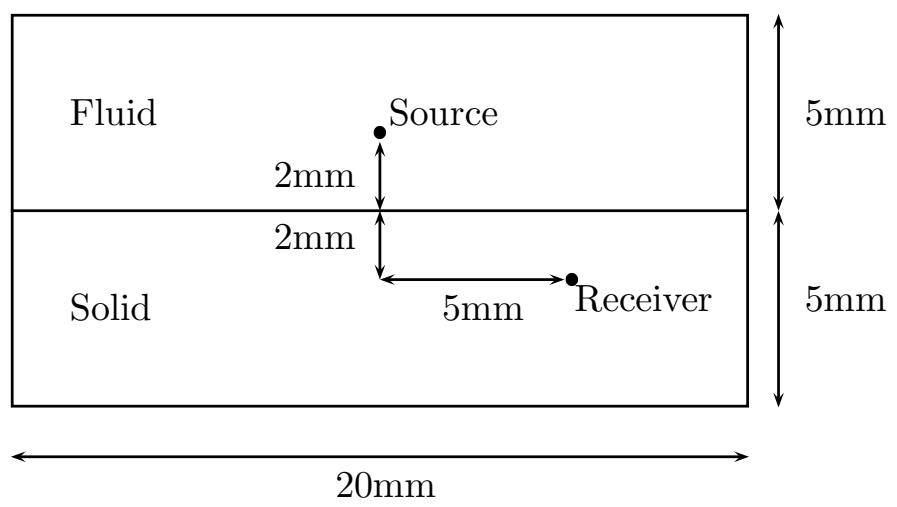

Fig. 9. Configuration of the experiment

- for the dual-dual methods we use $Q_{1}^{d i v}-Q_{0}$ finite elements, regular meshes in each domain but non-conforming : the space step is $0.1 \mathrm{~mm}$ in the fluid and $0.12 \mathrm{~mm}$ in the solid which corresponds to 15 points of calculation per wavelength in both fluid and 
solid (for S-wave). We use the same time step in the two grids chosen in order to satisfy CFL condition in both the fluid and the solid : $\Delta t=45 \mathrm{~ns}$;

- for the primal-primal method we use $Q_{5}$ finite elements. Here the global mesh is regular and conforming, the space step being equal to $1 \mathrm{~mm}$. Conversely we use different time step in the fluid $\left(\Delta t_{f}=35.6 \mathrm{~ns}\right)$ and in the solid $\left(\Delta t_{s}=17.8 \mathrm{~ns}\right)$ so that both CFL conditions are optimally satisfied.

We use two types of representation for the numerical results :

- snapshots at a given time (namely $t=4 \mu \mathrm{s}$ ) of the modulus of the velocity field (see figures 10, 11 and 12). The results presents a very good agreement between the three solutions ;

- to get a better idea on the accuracy, we also represent the variation in time of the two components of the velocity field at a receiver located inside the solid whose precise position is indicated in figure 9. The numerical solution obtained by the primal-primal method (the red curve) is compared to the analytical one (the blue curve) in figures 13 and 14 (we have represented the solution computed by the primal-primal method but the dual-dual one presents the same accuracy). The curves are perfectly superimposed, showing once again the accuracy of the method.

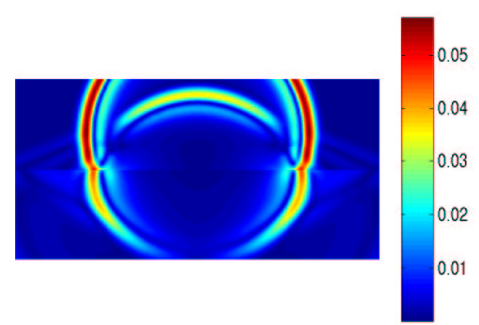

Fig. 10. Dual-dual formulation

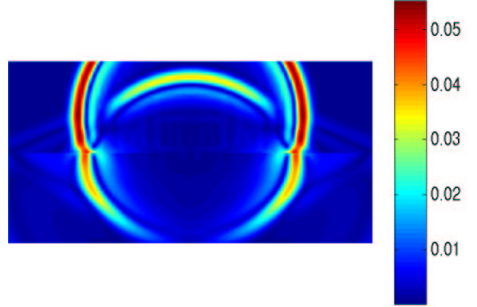

Fig. 11. Primal-primal formulation

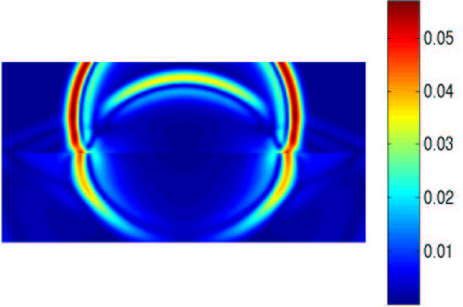

Fig. 12. Analytical Solutions

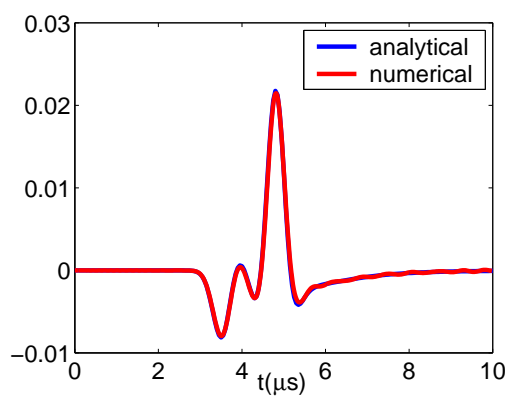

Fig. 13. The first component of the velocity

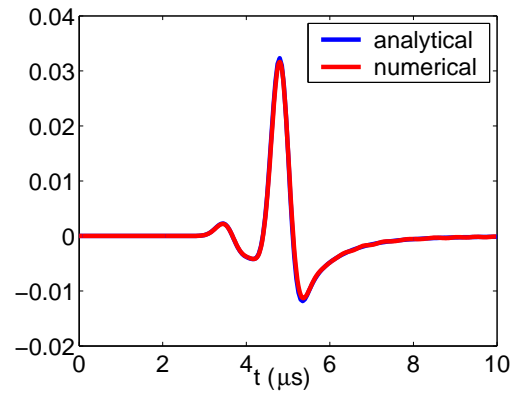

Fig. 14. The second component of the velocity 
In three dimensions. A similar experiment has been achieved in the case of two homogeneous half spaces in order to validate the primal-primal code with $Q_{5}$ finite elements in three dimensions (the three dimensions version of the dual-dual code is still not existing). The fluid medium is such that $c_{f}=1 \mathrm{~m} \mathrm{~s}^{-1}$ and $\rho_{f}=1 \mathrm{~kg} \mathrm{~m}^{-3}$ and the solid medium is such that $c_{S}=2 \mathrm{~m} \mathrm{~s}^{-1}, c_{P}=4 \mathrm{~m} \mathrm{~s}^{-1}$ and $\rho_{s}=1 \mathrm{~kg} \mathrm{~m}^{-3}$. The size of each domain is $10 \mathrm{~m} \times 5 \mathrm{~m} \times 10 \mathrm{~m}$. The source function in space is a point source, located in the fluid at $0.66 \mathrm{~m}$ from the interface - see figure 15 . The source function in time is the first derivative of a Gaussian whose dominating frequency is $1 \mathrm{~Hz}$.

This time, the same space step (conforming meshes) and the same time step have been

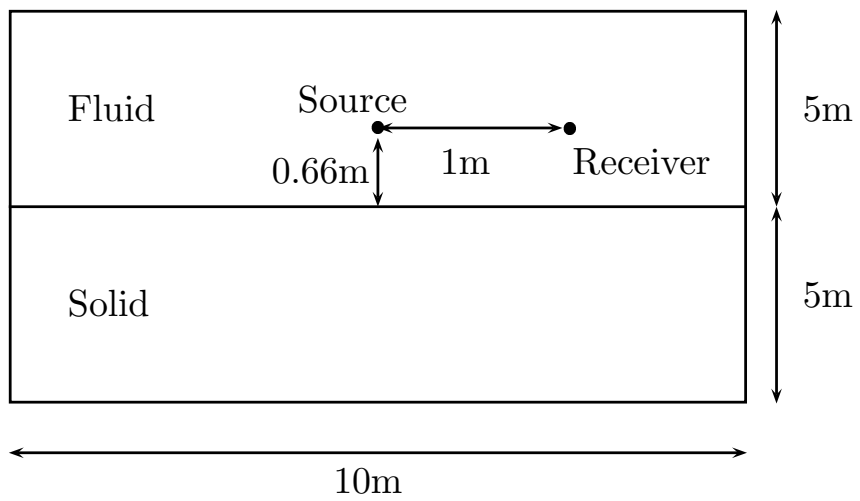

Fig. 15. Configuration of the experiment

chosen in the fluid and in the solid. Once again, to show that the coupling does not affect the accuracy, the space step is chosen by using the same criterion as for a single fluid or a single solid : $h=0.66 \mathrm{~m}$ and the time-step is chosen to satisfy the CFL condition in both the fluid and the solid : $\Delta t=9 \mathrm{~ms}$.

Snapshots of the modulus of the velocity field obtained by the numerical method at time $t=3 \mathrm{~s}$ are represented in figure 16. The analytical solution obtained by the Cagniard-de Hoop method is represented in figure 17. We also have computed the first and the second components of the velocity in the fluid along the time thanks to a receiver located as shown in figure 9. The numerical solution (the red curve) is compared to the analytical one (the blue curve) in figures 18 and 19. One observes a similar precision as the one obtained in two dimensions. The slight deterioration of the results after $t=3 \mathrm{~s}$ have to be attributed to the fact that the parameters into the PML domain have not been chosen sufficiently well.

\subsection{More realistic experiments}

The propagation of ultra-sounds through cortical bones, which is of particular interest for the diagnoses of osteoporosis ${ }^{7}$, can be modelized via a fluid-structure interaction problem. The 

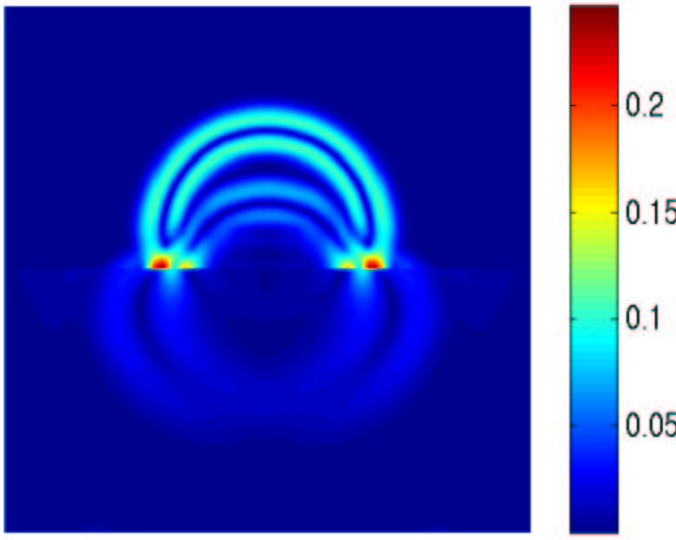

Fig. 16. Primal-primal formulation

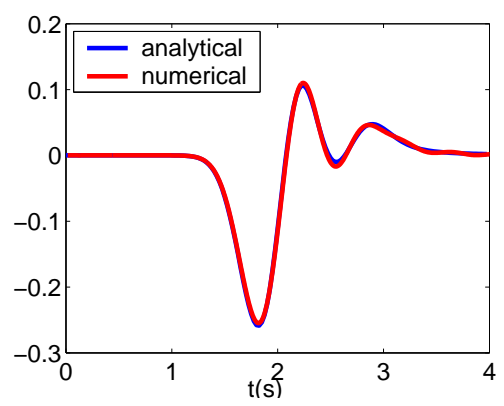

Fig. 18. The first component of the velocity

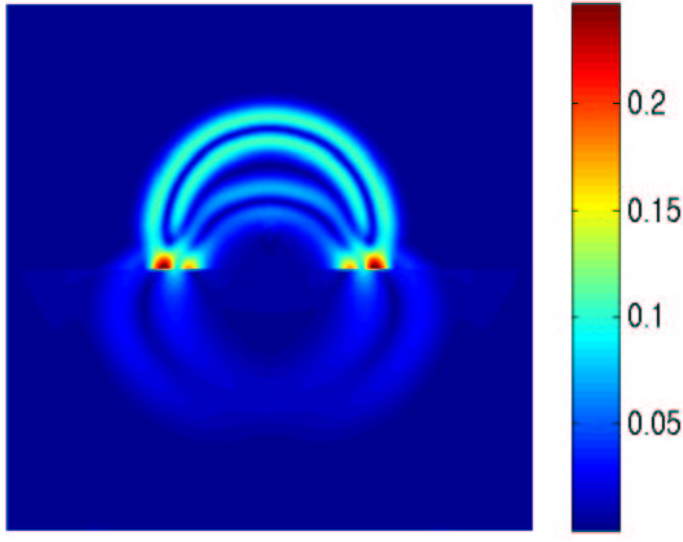

Fig. 17. Analytical Solution

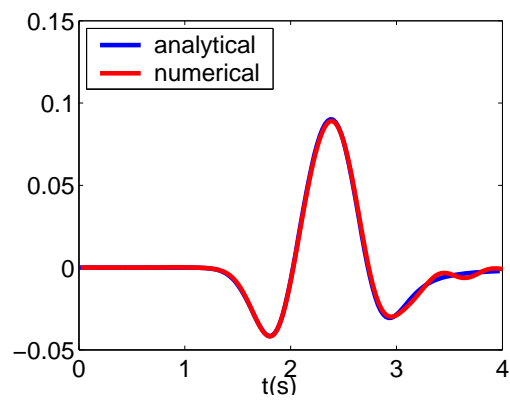

Fig. 19. The second component of the velocity

skin, the muscles and the marrow behaves merely like water while the bone behaves like an anisotropic solid medium. The following experiments are applications of the primal-primal method to such problems, first in two dimensions, then in three dimensions.

In two dimensions. We have first computed the propagation of a wave through a section of a bone. The figure 20 represents the configuration of the experiments :

- the inner disk is the marrow and is considered as filled with water $\left(c_{f}=1500 \mathrm{~m} \mathrm{~s}^{-1}\right.$ and $\rho_{f}=1000 \mathrm{~kg} \mathrm{~m}^{-3}$ ), its radius is $5 \mathrm{~mm}$;

- the circlet is the bone regarded as an isotropic solid with $c_{S}=1700 \mathrm{~m} \mathrm{~s}^{-1}, c_{P}=3400 \mathrm{~m} \mathrm{~s}^{-1}$ and $\rho_{s}=1850 \mathrm{~kg} \mathrm{~m}^{-3}$ (the anisotropy only concerns the third dimension). The external radius of the circlet is $10 \mathrm{~mm}$;

- the exterior medium that represents the muscles and the skin, is also assimilated to water.

- the computational domain is limited with the help of PML to simulate an infinite domain.

The mesh of this domain is represented in figure 21 . The source function in space is a line 


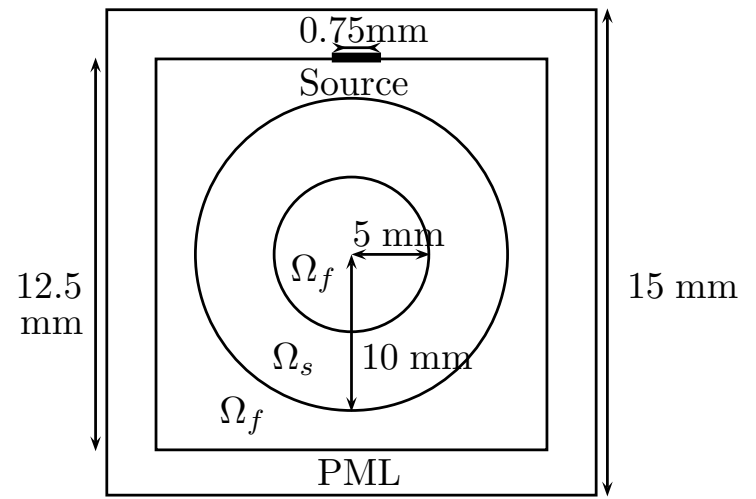

Fig. 20. Configuration of the experiment

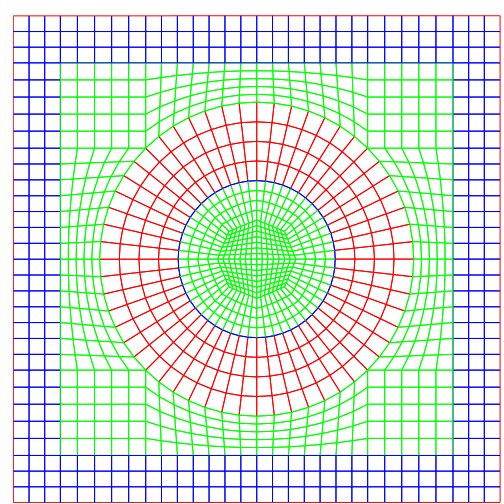

Fig. 21. Mesh

source parallel to the $\mathrm{x}$-axis whose center is located on the top edge of the green square and whose length is $0.75 \mathrm{~mm}$ (see figure 20). The source function in time is the first derivative of a Gaussian whose dominating frequency is $1 \mathrm{MHz}$. The time step is $9.5 \mu \mathrm{s}$ in the solid and $19 \mu$ s in the fluid. Curved elements are used to modelize the curved interface.

The modulus of the velocity is represented in figures $22,23,24$ and 25 respectively at time $t=2,4,6,8 \mathrm{~ms}$.

In three dimensions. The latter domain has been extruded to obtain a cylinder of height $30 \mathrm{~mm}$. The source in space is now a rectangle $12 \mathrm{~mm} \times 1 \mathrm{~mm}$ whose great and little edge are respectively parallel to the $\mathrm{x}$-axis and to the $\mathrm{z}$-axis. The coefficients of its elastic tensor are (in GPa) :

$$
C=\left[\begin{array}{cccccc}
21.5 & 11.5 & 11.5 & & & \\
21.5 & 11.5 & 11.5 & 0 & \\
21.5 & 11.5 & 29.6 & & \\
& & 6 & 0 & 0 \\
0 & & 0 & 6 & 0 \\
& & & 0 & 0 & 5
\end{array}\right]
$$

This corresponds to a medium which is isotropic in the $(x, y)$ plane (the same as in the $2 \mathrm{D}$ experiment) and anisotropic in the $(x, z)$ and $(y, z)$. Contrary to the latter experiment, we have used the same time-step $(8 \mu \mathrm{s})$ in both fluid and solid media. The result of the experiment are presented on the next page :

- figure 26 represents the modulus of the velocity at time $t=1.5,3,4.5,6 \mathrm{~ms}$ on a $3 \mathrm{D}$ view :

- figure 27 represents the modulus of the velocity at time $t=1.5,3,4.5,6 \mathrm{~ms}$ on a vertical 


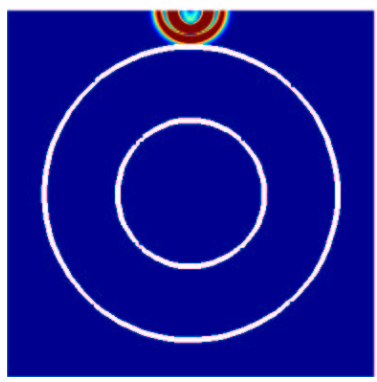

Fig. 22. $\mathrm{t}=2 \mathrm{~ms}$

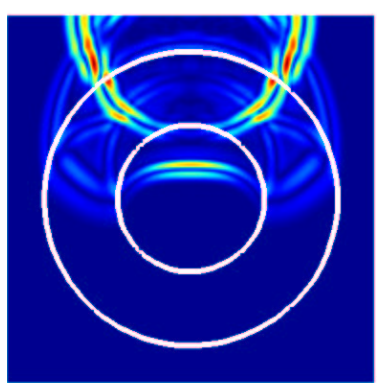

Fig. $24 . \mathrm{t}=6 \mathrm{~ms}$

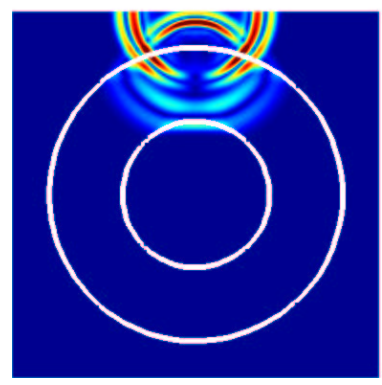

Fig. 23. $\mathrm{t}=4 \mathrm{~ms}$

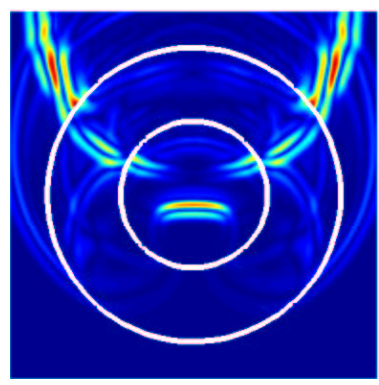

Fig. 25. $\mathrm{t}=8 \mathrm{~ms}$

section orthogonal to the source :

- figure 28 represents the modulus of the velocity at time $t=1.5,3,4.5,6 \mathrm{~ms}$ on an horizontal section :

- figure 29 represents the modulus of the velocity at time $t=1.5,3,4.5,6 \mathrm{~ms}$ on the external boundary of the solid cylinder.

Both 2D and 3D of numerical results (that have been compared - with a good agreement - to the solution obtained with finite difference $\operatorname{code}^{7}$ ) give a good idea of the complexity of wave propagation inside bones.

\section{References}

1. C. Tsogka, "Modélisation mathématique et numérique de la propagation des ondes élastiques tridimenssionnelles dans des milieux fissurés", Ph.D. thesis, Université Paris Dauphine-Paris IX, 1999.

2. S. Fauqueux, "Eléments finis spectraux et couches absorbantes parfaitement adaptées pour la propagation d'ondes élastiques en milieu transitoire", Ph.D. thesis, Université Paris DauphineParis IX, 2003.

3. P. Joly, "Variational Methods for Time-Dependent Wave Propagation Problems", in Topics in Computational Wave Propagation, Direct and Inverse Problems, LNCSE, 2003, pp 201-264.

4. O. Poncelet, "Réponse transitoire à une source impulsionnelle en espace en milieu anisotrope. Calcul de fonctions de Green par la méthode de Cagniard-de Hoop", in Ondes élastiques dans les solides, Cours de l'Ecole des ondes, Inria, 2001, pp 277-330. 

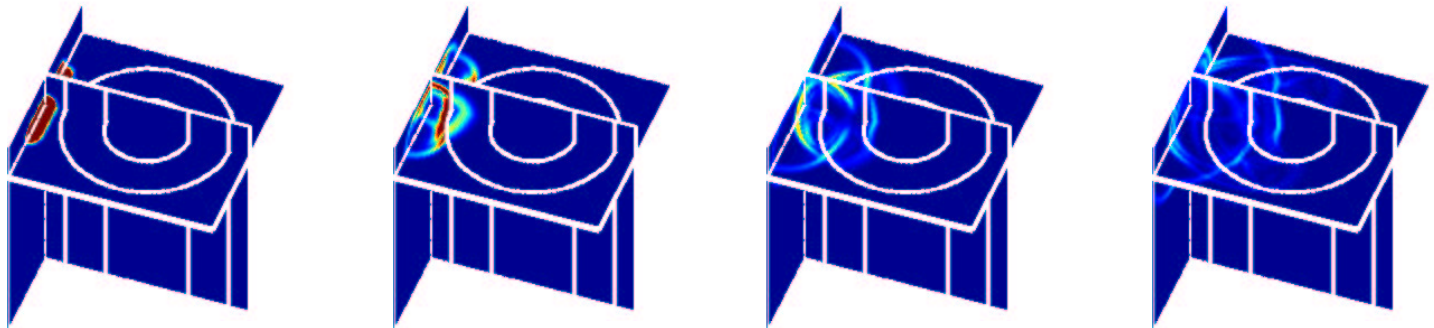

Fig. 26. The solution upon a $3 \mathrm{D}$ view
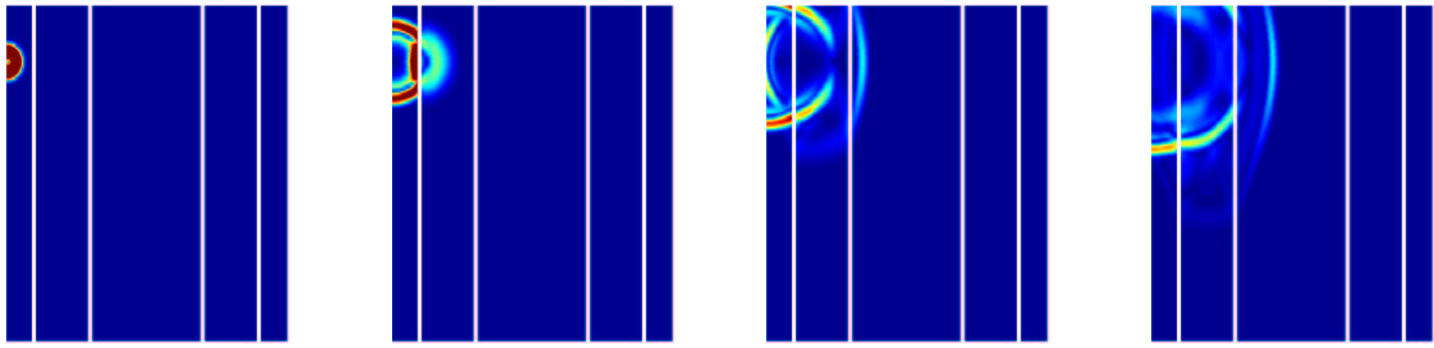

Fig. 27. The solution upon a vertical section
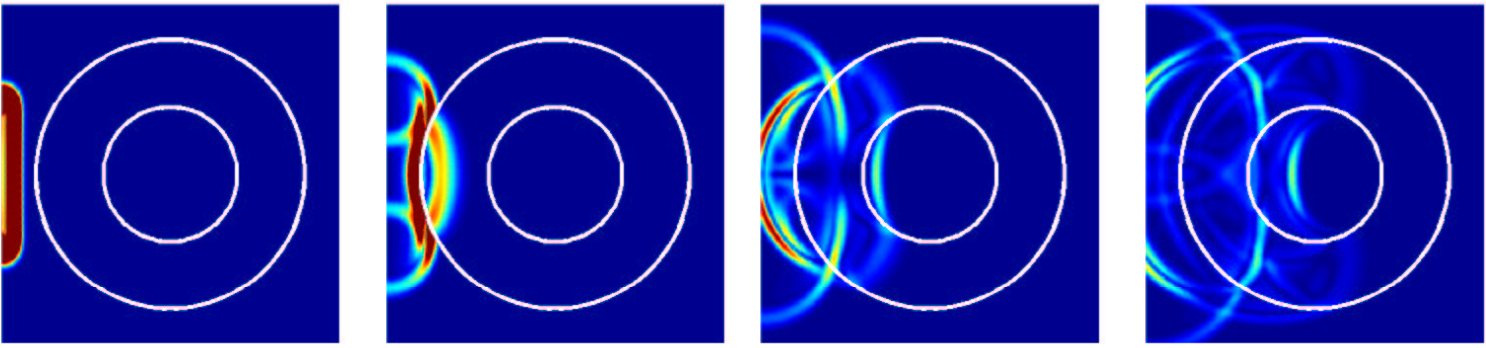

Fig. 28. The solution upon an horizontal section
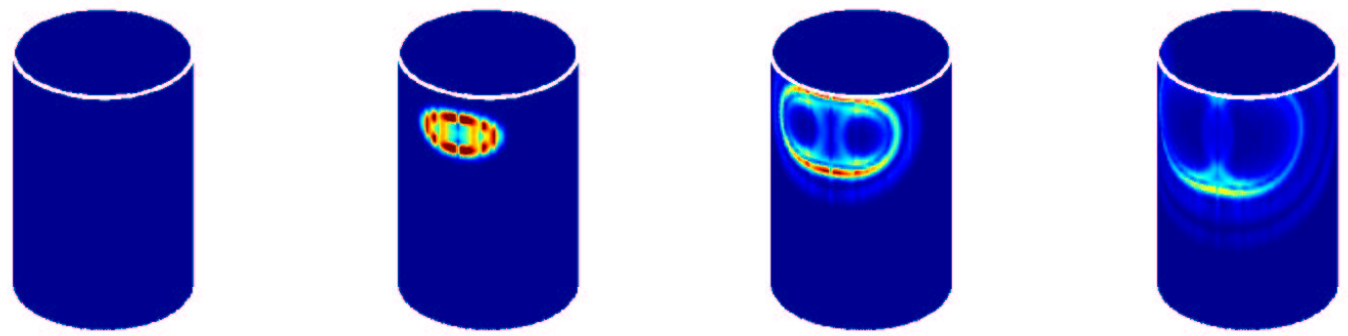

Fig. 29. The solution upon the external boundary of the cylinder

5. A. T. De Hoop, "The surface line source problem", Appl. Sci. Res. B, 8,1959, pp. 349-356.

6. J. Rodríguez, "Une Nouvelle Méthode de Raffinement de Maillage Spatio-Temporel pour l'Équation des Ondes ",C. R. Acad. Sci. Paris Sér. I Math., to be published.

7. E. Bossy, "Evaluation ultrasonore de l'os cortical par transmission axiale : modélisation et expérimentation in vitro et in vivo", Ph.D. thesis, Université Paris 6, 2003.

8. G. Derveaux "Modélisation numérique de la guitare acoustique", Ph.D. thesis, Ecole Polytechnique, 2002. 
9. J.M. Crolet and R. Ohayon (eds.), "Computational Methods for Fluid-Structure interaction". Pitman Research Notes in Mathematics 306, Longman, 1994.

10. M.A. Hamdi, Y. Ousset and G. Verchery, "A displacement method for the analysis of vibrations of coupled fluid-structure system" , Internat. J. Numer. Meth. Eng. 13, 1978, 139-150.

11. D. Komatisch, C. Barnes and J. Tromp, "Wave propagation near a fluid-solid interface : a spectral element approach", Geophysics, 65 2000, 623-63. 